\title{
Application of Arduino Programming Using ML8511 UV Sensor Hookup Guide to Learning the Effect of Ultraviolet's Level
}

\author{
Nugroho Adi Pramono a, *, Octavia Ghaisyani ${ }^{\text {b }}$, Bertha Anggita Purwandani ${ }^{\text {c }}$, Fauzan \\ Ibnu Sofyan d \\ ${ }^{a}$ Department of Physics, Universitas Negeri Malang \\ Malang, East Java, Indonesia
}

*Corresponding author's e-mail: nugroho.adi.fmipa@um.ac.id

\begin{abstract}
This paper learn programming of an Arduino. This also explores on how it can be used as a tool for study and research works. Learn how to program with Arduino, connect the project board to software on your computer, create sensor with the decision making and memory storing abilities of the Arduino. The ML8511 UV sensor outputs an analog signal in relation to the amount of UV light it detects and the effect to human body if get UV light in levels. The result are from three different locations, such as two outdoor and indoor start from 6.30 a.m until 10.45 a.m. Data shows that in outdoor during early morning or late afternoon hours the sun's rays pass at a greater angle through the atmosphere. Much more UV radiation is absorbed and less reaches the Earth, on the contrary in indoor UV index was 0 . UV levels are highest under cloudless skies, and cloud cover generally reduces a person's exposure. However, light or thin clouds have little effect and may even enhance UV levels because of scattering. Don't be fooled by an overcast day or a cool breeze! Even a long stay in open shade, for example between buildings, may give a sensitive person a sunburn on a day with high UV levels. Therefore, it is important to know effect of ultraviolet's level, from application of Arduino Programming using ML8511 UV sensor simplify the research.
\end{abstract} Keywords: Sensor UV; ML8511; Arduino; UV lights; Software.

\section{Introduction}

The Electromagnetic (EM) Spectrum is simply a name scientists use when referring to the entire range of radiation types. Radiation is energy that travels and spreads out as it moves. Examples include visible light and radio waves. Other examples of EM radiation are ultraviolet and infrared light, microwaves, X-rays and gamma-rays. Ultraviolet radiation is that part of the electromagnetic spectrum between visible light and x-rays. UV radiation is classified into three primary types: 
ultraviolet A (UVA), ultraviolet B (UVB), and ultraviolet C (UVC). These groups are based on the measure of their wavelength. UVA, (know as longwave or blacklight), is radiation with wavelengths between $315 \mathrm{~nm}$ and 400nm. UVB ( midrange) spans wavelengths from 280 to $315 \mathrm{~nm}$ and UVC (shortwave) covers $280 \mathrm{~nm}$ down to about 30nm [2]. Ultraviolet light is absorbed strongly by living systems with the result that its penetration is relatively superficial, and when we deal even with a quite thin layer the amount of light reaching different levels may vary greatly [1]. All the UVC and most of the UVB radiation is absorbed by the earth's ozone layer, so nearly all of the ultraviolet radiation received on earth is UVA. UVA and UVB radiation can both affect health. Even tough UVA radiation is weaker than UVB, its penetrates deeper into the skin and is more constant throughout the year. Since UVC radiation is absorbed by the earth's ozone layer, it does not pose as much of a risk.

In this research, will introduce you to the use of Arduino with project board as a sensor data reader of ultraviolet light. we will build a UV meter using the Arduino and the ultraviolet sensor (ML8511) with a LCD display as the display for the meter. The LCD is used to display the UV index which is an international standard unit for the intensity of ultraviolet rays from the sun being experienced in a particular place and at a particular time. It can be seen from the chart that when UV level is extremely high it is advised to stay indoors, the reason for this is, the UV index indoors is mostly zero. This means that when testing the device we are about to build, you should take it outside as shown in the image below, as the value won't change if you test indoors. The ML8511 sensor is very easy to use. It outputs a analog voltage that is linearly related to the measured UV intensity $(\mathrm{mW} / \mathrm{cm} 2)$. If your microcontroller can do an analog to voltage conversion, then you can detect the level of UV.

Global heating temperature is caused by excessive carbon monoxide and not balanced with green open areas especially in urban areas that minimize urban parks, This causes poor air quality and, most of all, the depletion of the ozone layer in the atmosphere. The function of the ozone layer is to filter out the exposure of harmful UV radiation into the earth's surface, this circumstance should be a concern to general public because it can interfere with health so people should also know the amount of carbon monoxide and UV radiation in the place where the community is active. To be able to provide information and warning to the general public then made a system of $\mathrm{UV}$ and $\mathrm{CO}$ radiation detection in the air by using microcontroller Arduno. The design using the sensor ML8511 as a UVsensor. 


\section{Theory Basis}

a. Ultraviolet light

Ultraviolet radiation's (UV) placement in the electromagnetic spectrum [4]. The frequency of UV radiation lies between that of x-rays and visible light. UV has a higher frequency and shorter wavelength than visible light, and it has a lower frequency and longer wavelength than $\mathrm{X}$-radiation [3]. UV with its longer wavelength and less energy is less penetrating than X-ray and is sometimes absorbed.

Ultraviolet are classified as UVA, UVB, and UVC. UVA is found in the region between 320 and $400 \mathrm{~nm}$ and is the least powerful wavelength band of UV radiation [3]. UVA acts primarily to cause the melanin pigments in the skin to oxidize (darken) creating the cosmetic tan and has limited power to cause erythema[2]. UVB is found in the region between 280 and $320 \mathrm{~nm}$ [2]. It comprises the wavelengths primarily associated with erythema (sunburn), is also necessary for the production of vitamin $\mathrm{D}$ in the skin and is primarily responsible for stimulating increased melanin production. UVB wavelengths (at $305 \mathrm{~nm}$ ) have 1,000 times more erythemal power than UVA wavelengths. UVC is found in the region between approximately $200-280 \mathrm{~nm}$ and is called germicidal UV because of its proven effectiveness in killing sing-cell organisms[2]. Solar radiation in the UVC range is absorbed almost entirely by the atmosphere and that is fortunate considering that even a short overexposure to UVC is very harmful to the eyes and causes severe erythema (sunburn) [3]. b. Arduino

Arduino is an open-source electronics platform based on easy-to-use hardware and software. Arduino boards are able to read inputs - light on a sensor, a finger on a button, or a Twitter message - and turn it into an output - activating a motor, turning on an LED, publishing something online.

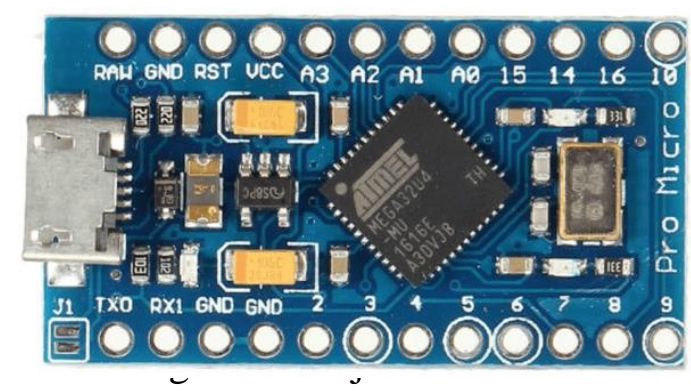

The Pro Micro is a ATmega32U4 open source development board[Figure 1]. The USB transceiver inside the $32 \mathrm{U} 4$ allows us to add USB connectivity on-board and do away with bulky external USB interface.

\section{c. Python}

Python is a widely used general-purpose, high level programming language. It was created by Guido van Rossum in 1991 and further developed by the Python Software Foundation. It was designed with an emphasis on code readability, and its syntax allows programmers to express their 
concepts in fewer lines of code. Python is a programming language that lets you work quickly and integrate systems more efficiently.

\section{Research Method}

The design of system ultraviolet light detection uses several tules, for example UV Sensor, Arduino as a sensor data, and OLED Display as an output. The detection system design chart is shown in Figure 2.

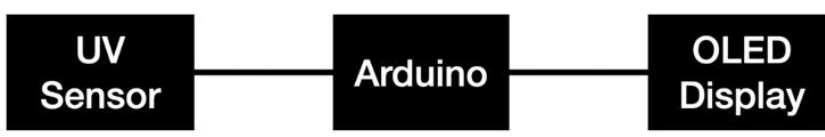

Figure 2. Design of an ultraviolet detection system

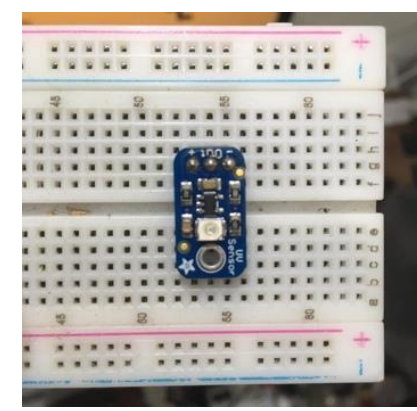

Figure 3. UV Sensor type GUVA-S12SD

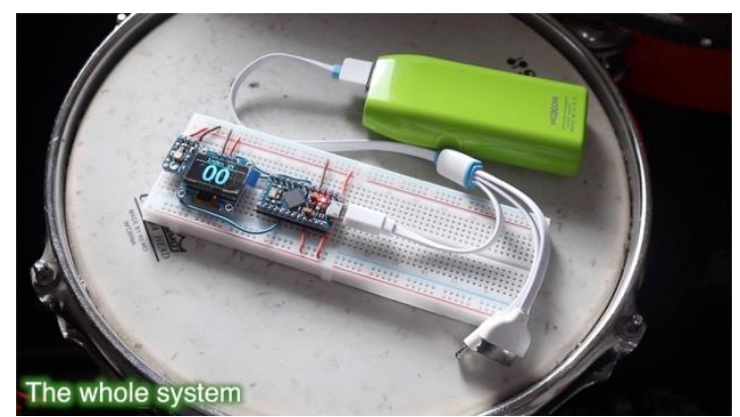

Figure 4. The whole system ultraviolet detection

Figure 3 is UV sensor type GUVA-21SD connected to charging-discharging circuit as an Analog to Digital Converter (ADC). After all connected, the Arduino as a sensor will read the data and result will be shown on tle OLED Display. 

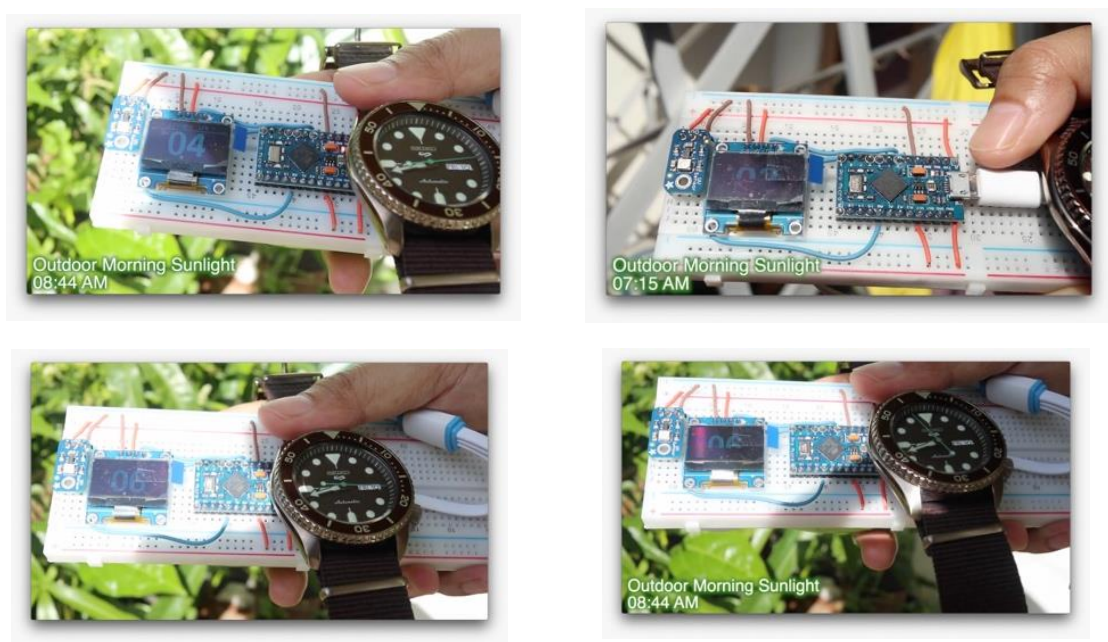

Figure 5. Result data

Figure 5 shown result data from the research that I have done. Before the Arduino can read the data, The data obtained from the UV sensor will be processed using the python programming language.

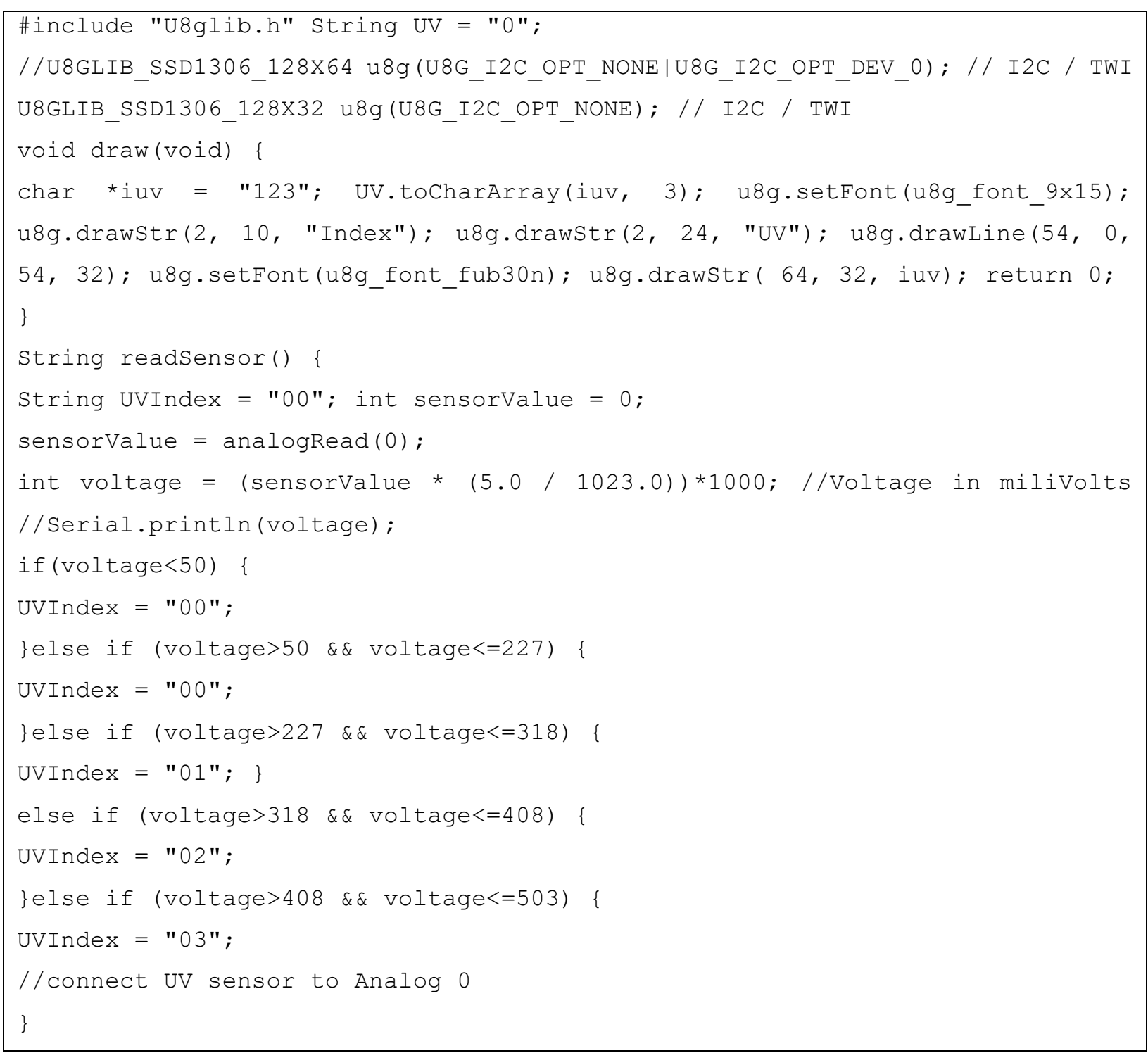




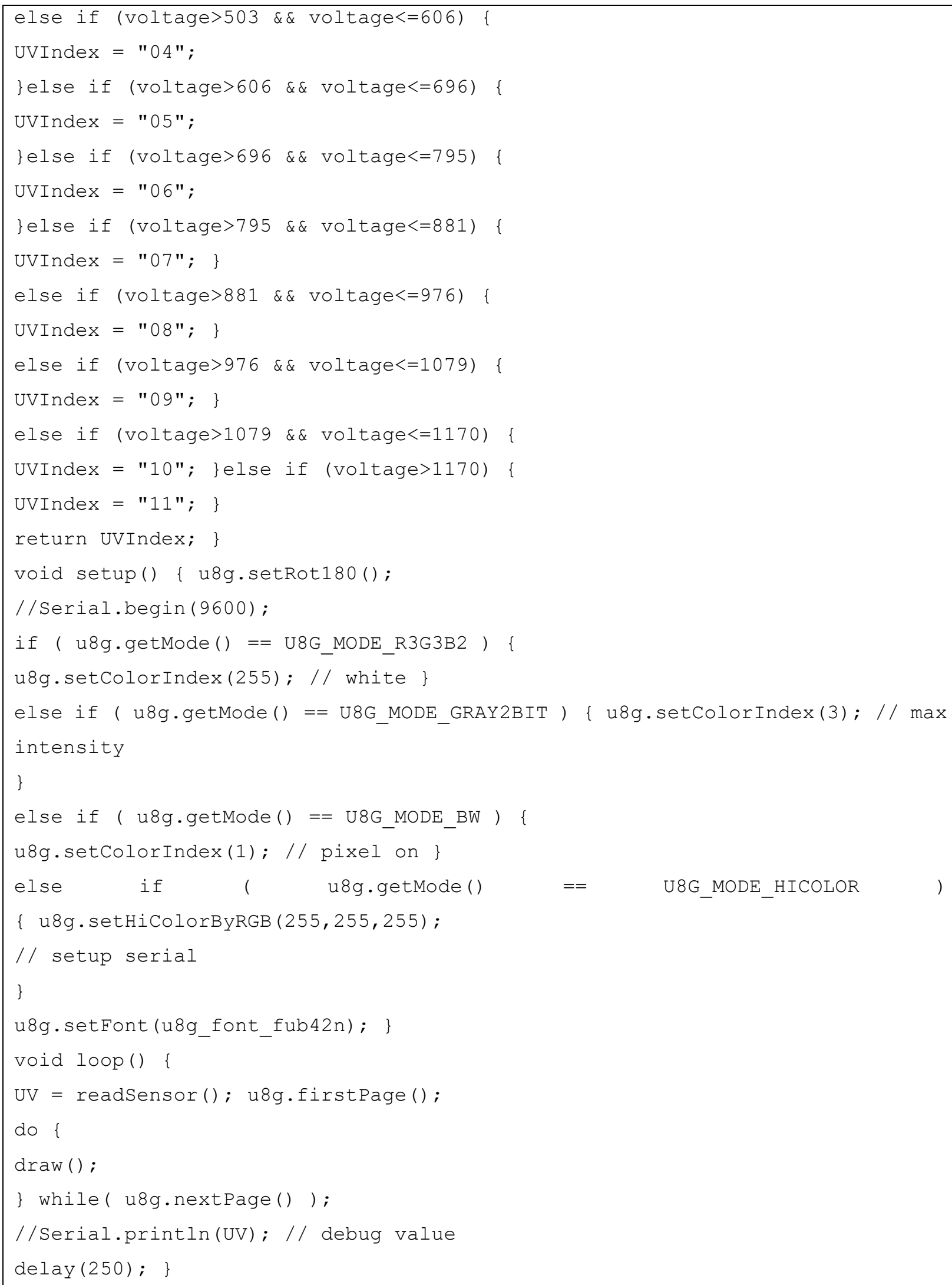




\section{Results and Discussion}

Tabel 1 .

UV index results

\begin{tabular}{cccc}
\hline & \multicolumn{3}{c}{ Index UV } \\
\cline { 2 - 4 } Time (a.m.) & A & B & Indoor \\
\hline $\mathbf{0 6 . 3 0}$ & 1 & 0 & 0 \\
$\mathbf{0 7 . 1 5}$ & 2 & 1 & 0 \\
$\mathbf{0 8 . 4 4}$ & 4 & 3 & 0 \\
$\mathbf{1 0 . 0 0}$ & 5 & 5 & 0 \\
$\mathbf{1 0 . 4 5}$ & 6 & 5 & 0 \\
\hline
\end{tabular}

$V_{0}=4.3 *$ Diode - Current - in $-u A \quad$ index $=\frac{V_{0}}{0.1} V$

To find out the UV index we use two different locations, location A is at coordinates -7.973731, 112.579935 and location B is at coordinates $-7.9606839,112.6191241$. first of all calibrate system with . From the research data from 2 outdoor locations and indoor locations, it can be seen that; from 06.30 a.m to 07.15 the category of ultraviolet light is still low with a UV index between $0-2$ from 08.44 a.m to 10.00 a.m, the detected ultraviolet light was in the moderate category with a UV index of 6 and ultraviolet light at location B was categorized as moderate with a UV index 5. In indoor locations, it was known that the UV index was 0 . this is due to the results of rounding . A number from 0-0.99 is considered to be indexed zero. The difference in UV index result is caused by several factors, namely location, surrounding environment, and weather. Based on these result, light can be reflected on the earth's surface because only a little UV light reaches the earth's surface. From the result system shown that Application Of Arduino Programming Using ML8511 UV Sensor Hookup Guide To Learning the Effect Of Ultraviolet's Level was not optimal seeing in indoor UV index not detected.

\section{Conclusion}

Based on the research data, it can be concluded that the design of an ultraviolet light detection system using the Arduino for learning about ultraviolet light reflection on the earth's surface has been successfully carried out provided that the placement of the UV index measuring instrument is exposed to direct sunlight and is in accordance with the UV index data from BMKG as a reference. From the results of the UV index obtained, it can also be seen that ultraviolet rays that reach the earth can be reflected even though they are imperfect, because the ozone layer on the earth's surface is damaged and depleted quite severely. 


\section{References}

[1] H.F Blum, carcinogenesis by Ultraviolet Light (Princeton University Press, New Jersey, 2016) pp 17-36

[2] M. P. Brown and K. Austin, Appl. Phys. Letters 85, 2503-2504 (2004).

[3] B. R. Jackson and T. Pitman, U.S. Patent No. 6,345,224 (8 July 2004)

[4] Parrish,John. UV-A: Biological Effects of Ultraviolet Radiation with Emphasis on Human Respones to Longwave Ultraviolet (Springer US, New York, Copyright 1978) 